Journal of Mathematics and Statistics 6 (1): 1-3, 2010

ISSN 1549-3644

(C) 2010 Science Publications

\title{
Transient Solution to an infinite Server Queue with Varying Arrival and Departure Rate
}

\author{
A.A. El-Sherbiny \\ Department of Mathematics, Faculty of Science, Menoufia University, Menoufia, Egypt
}

\begin{abstract}
Problem statement: In many potential application of queueing theory, the transient solution of queueing system is important. Approach: This study presented the transient solution for infinite server queues with Poisson arrivals and exponential service times when the parameters of both distributions are allowed to vary with time. Based on generating functions technique which results in a simple differential equation. Using the properties of Bessel functions in the solution of this differential equation, the solution of an infinite server queues can be given in simple form. Results: The researcher obtained the transient solution an infinite server queues with Poisson arrivals and exponential service times when the parameters of both distributions are allowed to vary with time and prove that some past results are special case from his results. Conclusion: These results indicated that the probabilities can be extracted in a direct way.
\end{abstract}

Key words: Transient solution, infinite server queue, modified Bessel function, generating functions

\section{INTRODUCTION}

The queue size distribution for the $\mathrm{M} / \mathrm{M} / \infty$ queue, when the Poisson processes are time homogeneous, is given in most texts on queueing theory. Saaty (1961) derived the queue size distribution when input is not time homogeneous and showed that if the departure rate is constant and the initial queue size is Poisson, then the resulting queue size distribution is Poisson with a timedependent parameter.

Collings and Stoneman (1976) have been shown that the same result holds for a time-dependent departure rate and they derived the queue size distribution for the same problem in the form of a probability generating function.

Abol'nikov (1968) allowed requests for service to come in batches of varying size, the instants of arrival of the requests following a non-homogeneous Poisson process. The generating function of the queue size distribution is derived at any point in time.

Shanbhag (1966) considered the same system but removes the restriction that the service time distribution must be exponential. Moreover, the results for a general service time distribution are obtained and if the requests come singly (one at time), then queue size distribution is always Poisson when the queue is empty initially (Shanbhag, 1966).

Clarke (1956) studied queues resulting from nonhomogenous Poisson processes and provided a complete theoretical solution to the single-server case. Leese and Boyd (1966) gave a useful discussion of the numerical methods that have proposed. The most simple and direct method of solution would be to solve the birth and death equations by a normal numerical method.

Bagchi and Templeton (1972) applied the queues resulting in homogeneous Poisson processes, their method is not applicable to the more general forms of time-depending.

Most works concerning non-homogeneous Poisson Processes have been concerned with the queue size distribution, but Hasofer (1964) described a method of obtaining the waiting time distribution for the singleserver case, using an approach based on Takacs (1955).

Problem formulation: The birth and death equations for an infinite server queue are:

$$
\begin{aligned}
\mathrm{P}_{0}^{\prime}(\mathrm{t})= & -\lambda(\mathrm{t}) \mathrm{P}_{0}(\mathrm{t})+\mu(\mathrm{t}) \mathrm{P}_{1}(\mathrm{t}) \\
\mathrm{P}_{\mathrm{n}}^{\prime}(\mathrm{t})= & \lambda(\mathrm{t}) \mathrm{P}_{\mathrm{n}-1}(\mathrm{t})-(\lambda(\mathrm{t})+\mathrm{n} \mu(\mathrm{t})) \mathrm{P}_{\mathrm{n}}(\mathrm{t}) \\
& +(\mathrm{n}+1) \mu(\mathrm{t}) \mathrm{P}_{\mathrm{n}+1}(\mathrm{t}), \mathrm{n}>0
\end{aligned}
$$

In this study, the researcher restricts their attention to the special case the following initially units in the system:

$$
\lambda(\mathrm{t})=\lambda \mathrm{t}^{\mathrm{m}+1} \mathrm{e}^{-\mathrm{mt}} \text { and } \mu(\mathrm{t})=\mu \mathrm{t}^{\mathrm{m}+1} \mathrm{e}^{-\mathrm{mt}}, \mathrm{m}=0,1,2, \ldots, \mathrm{n}
$$

Define:

$$
\begin{aligned}
& \mathrm{q}_{\mathrm{n}}(\mathrm{t})=\mathrm{e}^{(\lambda+\mathrm{n} \mu) \mathrm{t}}\left[\mathrm{t}^{\mathrm{m}+1} \mathrm{e}^{-\mathrm{mt}}\left(\mathrm{n} \mu \mathrm{P}_{\mathrm{n}}(\mathrm{t})-\lambda \mathrm{P}_{\mathrm{n}-1}(\mathrm{t})\right)\right], \\
& \mathrm{n}=1,2, \ldots ; \mathrm{t}>0
\end{aligned}
$$


And consider:

$$
H(z, t)=\sum_{n=-\infty}^{\infty} q_{n}(t) z^{n}
$$

Differentiating (3) and (4) with respect to $t$ and using (1) and (2), we get:

$$
\frac{\partial H(z, t)}{\partial t}=\left(\lambda z+\frac{n \mu}{z}\right) H(z, t)+G\left(H(z, t), \frac{\partial H(z, t)}{\partial z}\right)
$$

Where:

$$
\begin{aligned}
& \mathrm{G}\left(\mathrm{H}(\mathrm{z}, \mathrm{t}), \frac{\partial \mathrm{H}(\mathrm{z}, \mathrm{t})}{\partial \mathrm{z}}\right) \\
& =-\left(\lambda \mathrm{z}+\frac{\mathrm{n \mu}}{\mathrm{z}}\right) \mathrm{H}(\mathrm{z}, \mathrm{t}) \\
& +\left(\begin{array}{l}
\lambda-\mathrm{m}+\frac{\mathrm{m}+1}{\mathrm{t}}-\mu \mathrm{t}^{\mathrm{m}+1} \frac{\mathrm{e}^{-(\mathrm{m}+\mu \mathrm{t})}}{\mathrm{z}}-\lambda \mathrm{t}^{\mathrm{m}+1} \mathrm{e}^{-\mathrm{mt}} \\
+\lambda \mathrm{zt}^{\mathrm{m}+1} \mathrm{e}^{-(\mathrm{m}-\mu) \mathrm{t}}
\end{array}\right) \mathrm{H}(\mathrm{z}, \mathrm{t}) \\
& +\left(\mu \mathrm{z}-\mu \mathrm{zt}^{\mathrm{m}+1} \mathrm{e}^{-\mathrm{mt}}+\mu \mathrm{t}^{\mathrm{m}+1} \mathrm{e}^{-(\mathrm{m}+\mu \mathrm{t})}\right) \cdot \frac{\partial \mathrm{H}(\mathrm{z}, \mathrm{t})}{\partial \mathrm{z}}
\end{aligned}
$$

The resulting in (5) is a linear differential equation in $\mathrm{H}(\mathrm{z}, \mathrm{t})$ and its solution is given by:

$$
\begin{aligned}
& H(z, t) \cdot \exp \left[-\left(\lambda z+\frac{n \mu}{z}\right) t\right] \\
& =\int_{1}^{t} G(u) \exp \left[\left(\lambda z+\frac{n \mu}{z}\right)(t-u)\right] \cdot d u+C
\end{aligned}
$$

$$
\text { Put } \mathrm{t}=1 \text {, then: }
$$

$$
C=\exp \left[-\left(\lambda z+\frac{n \mu}{z}\right)\right] H(z, 1)
$$

And:

$$
H(z, 1)=z^{a}\left[\begin{array}{l}
\left(a \mu e^{(\lambda+a \mu-m)}-\lambda z e^{(\lambda+(a+1) \mu-m)}\right) \\
\left(1-\delta_{1 a}\right)-\lambda e^{(\lambda+\mu-m)} \cdot \delta_{1 a}
\end{array}\right]
$$

Whence:

$$
\begin{aligned}
H(z, t)= & \exp \left[\left(\lambda z+\frac{n \mu}{z}\right)(t-1)\right] \cdot z^{a} \\
& {\left[\begin{array}{l}
\left(a \mu e^{(\lambda+a \mu-m)}-\lambda z e^{(\lambda+(a+1) \mu-m)}\right) \\
\left(1-\delta_{1 a}\right)-\lambda z e^{(\lambda+\mu-m)} \delta_{1 a}
\end{array}\right] } \\
& +\int_{1}^{t} G(u) \exp \left[\left(\lambda z+\frac{n \mu}{z}\right)(t-u)\right] \cdot d u
\end{aligned}
$$

But it is know that:

$$
\exp \left\{\left(\lambda z+\frac{\mathrm{n} \mu}{\mathrm{z}}\right) \mathrm{t}\right\}=\sum_{\mathrm{n}=-\infty}^{\infty}(\mathrm{vz})^{\mathrm{n}} I_{\mathrm{n}}(\mathrm{rt})
$$

With:

$$
r=2 \sqrt{n \lambda \mu} \text { and } v=\sqrt{\lambda / n \mu}
$$

Then:

$$
\begin{aligned}
H(z, t)= & \sum_{n=-\infty}^{\infty}(v z)^{n} I_{n}(r(t-1)) \cdot z^{a} \\
& {\left[\begin{array}{l}
\left(a \mu e^{(\lambda+a \mu-m)}-\lambda z e^{(\lambda+(a+1) \mu-m)}\right) \\
\left(1-\delta_{1 a}\right)-\lambda z e^{(\lambda+\mu-m)} \delta_{1 a}
\end{array}\right] } \\
& +\int_{1}^{t} G(u) \sum_{n=-\infty}^{\infty}(v z)^{n} I_{n}(r(t-u)) \cdot d u
\end{aligned}
$$

where, $\mathrm{I}_{\mathrm{n}}($.$) is the modified Bessel function. Comparing$ the coefficients of $\mathrm{z}^{\mathrm{n}}$ on both sides for $\mathrm{n} \geq 1$, then:

$$
\begin{aligned}
\mathrm{q}_{\mathrm{n}}(\mathrm{t})= & a \mu \mathrm{e}^{(\lambda+\mathrm{au}-\mathrm{m})}\left(1-\delta_{1 \mathrm{a}}\right) \mathrm{v}^{\mathrm{n}-\mathrm{a}} \mathrm{I}_{\mathrm{n}-\mathrm{a}}(\mathrm{r}(\mathrm{t}-1)) \\
& -\lambda \mathrm{e}^{(\lambda+(\mathrm{a}+1) \mu-\mathrm{m})}\left(1-\delta_{\mathrm{la}}\right) \mathrm{v}^{\mathrm{n}-\mathrm{a}-1} \mathrm{I}_{\mathrm{n}-\mathrm{a}-1}(\mathrm{r}(\mathrm{t}-1)) \\
& -\lambda \mathrm{e}^{(\lambda+\mu-\mathrm{m})} \delta_{1 \mathrm{a}} \mathrm{v}^{\mathrm{n}-\mathrm{a}-1} \mathrm{I}_{\mathrm{n}-\mathrm{a}-1}(\mathrm{r}(\mathrm{t}-1))+\int_{1}^{\mathrm{t}} \mathrm{G}(\mathrm{u}) \mathrm{v}^{\mathrm{n}} \mathrm{I}_{\mathrm{n}} \\
& (\mathrm{r}(\mathrm{t}-\mathrm{u})) \cdot \mathrm{du}
\end{aligned}
$$

Since $\mathrm{q}_{\mathrm{n}}(\mathrm{t})=0$ for $\mathrm{n}<0$ and using $\mathrm{I}_{\mathrm{n}}(\mathrm{u})=\mathrm{I}_{\mathrm{n}}(\mathrm{u})$ Eq. 10 becomes:

$$
\begin{aligned}
& \int_{1}^{t} G(u) \sum_{n=-\infty}^{\infty}(v z)^{n} I_{n}(v) \cdot d u \\
& =-a \mu e^{(\lambda+a \mu-m)}\left(1-\delta_{1 a}\right) v^{n-a} I_{n+a}(r(t-1))+\lambda e^{(\lambda+(a+1) \mu-m)} \\
& \left(1-\delta_{1 a}\right) v^{n-a-1} I_{n+a+1}(r(t-1))-\lambda e^{(\lambda+\mu-m)} \delta_{1 a} v^{n-a-1} I_{n+a+1} \\
& (r(t-1))
\end{aligned}
$$

Where:

$$
\delta_{1 a}= \begin{cases}1, & a=0 \\ 0, & a \neq 0\end{cases}
$$

Using (11) in (10) for $\mathrm{n}=1,2, \ldots$, then:

$$
\begin{aligned}
\mathrm{q}_{\mathrm{n}}(\mathrm{t})= & a \mu \mathrm{e}^{(\lambda+\mathrm{a} u-\mathrm{m})}\left(1-\delta_{1 \mathrm{a}}\right) \mathrm{v}^{\mathrm{n}-\mathrm{a}}\left[\mathrm{I}_{\mathrm{n}-\mathrm{a}}(\mathrm{r}(\mathrm{t}-1))-\mathrm{I}_{\mathrm{n}+\mathrm{a}}\right. \\
& (\mathrm{r}(\mathrm{t}-1))]-\lambda \mathrm{e}^{(\lambda+(\mathrm{a}+1) \mu-\mathrm{m})}\left(1-\delta_{\mathrm{la}}\right) \mathrm{v}^{\mathrm{n}-\mathrm{a}-1} \\
& {\left[\mathrm{I}_{\mathrm{n}-\mathrm{a}-1}(\mathrm{r}(\mathrm{t}-1))-\mathrm{I}_{\mathrm{n}+\mathrm{a}+1}(\mathrm{r}(\mathrm{t}-1))\right]-\lambda \mathrm{e}^{(\lambda+\mu-\mathrm{m})} \delta_{1 \mathrm{a}} } \\
& \mathrm{v}^{\mathrm{n}-\mathrm{a}-1}\left[\mathrm{I}_{\mathrm{n}-\mathrm{a}-1}(\mathrm{r}(\mathrm{t}-1))-\mathrm{I}_{\mathrm{n}+\mathrm{a}+1}(\mathrm{r}(\mathrm{t}-1))\right]
\end{aligned}
$$


From (3) and by iteration method one can get:

$$
P_{n}(t)=\frac{\rho^{n}}{n !} P_{0}(t)+\frac{e^{m t}}{\mu t^{m+1}} \cdot \sum_{k=1}^{n} q_{k}(t) \cdot e^{-(\lambda+k \mu) t} \cdot \frac{(k-1) ! \rho^{n-k}}{n !}
$$

Substitute the value of $\mathrm{q}_{\mathrm{n}}(\mathrm{t})$ from (12) in (13), then the value of $\mathrm{P}_{0}(\mathrm{t})$ is:

$$
P_{0}(t)=\int_{1}^{t} q_{1}(u) e^{-(\lambda+\mu)} \cdot d u+\delta_{1 a}
$$

Special case: Let $m=0$, the results of $A 1$ Seedy and $A L$ Ibraheem (2003) are get as:

$$
P_{n}(t)=\frac{\rho^{n}}{n !} P_{0}(t)+\frac{1}{\mu t} \sum_{k=1}^{n} q_{k}(t) \cdot e^{-(\lambda+k \mu) t} \cdot \frac{(k-1) ! \rho^{n-k}}{n !}
$$

Where:

$$
\begin{aligned}
\mathrm{P}_{0}(\mathrm{t})= & \int_{1}^{\mathrm{t}} \mathrm{q}_{1}(\mathrm{u}) \mathrm{e}^{-(\lambda+\mu)} \cdot \mathrm{du}+\delta_{1 \mathrm{a}} \\
\mathrm{q}_{\mathrm{n}}(\mathrm{t})= & \mathrm{a} \mu \mathrm{e}^{(\lambda+\mathrm{a} \mu)}\left(1-\delta_{1 \mathrm{a}}\right) \mathrm{v}^{\mathrm{n}-\mathrm{a}}\left[\mathrm{I}_{\mathrm{n}-\mathrm{a}}(\mathrm{r}(\mathrm{t}-1))-\mathrm{I}_{\mathrm{n}+\mathrm{a}}(\mathrm{r}(\mathrm{t}-1))\right] \\
& -\lambda \mathrm{e}^{(\lambda+(\mathrm{a}+1) \mu)}\left(1-\delta_{1 \mathrm{a}}\right) \mathrm{v}^{\mathrm{n}-\mathrm{a}-1}\left[\mathrm{I}_{\mathrm{n}-\mathrm{a}-1}(\mathrm{r}(\mathrm{t}-1))-\mathrm{I}_{\mathrm{n}+\mathrm{a}+1}\right. \\
& (\mathrm{r}(\mathrm{t}-1))]-\lambda \mathrm{e}^{(\lambda+\mu)} \delta_{1 \mathrm{a}} \mathrm{v}^{\mathrm{n}-\mathrm{a}-1}\left[\mathrm{I}_{\mathrm{n}-\mathrm{a}-1}(\mathrm{r}(\mathrm{t}-1))\right. \\
& \left.-\mathrm{I}_{\mathrm{n}+\mathrm{a}+1}(\mathrm{r}(\mathrm{t}-1))\right]
\end{aligned}
$$

\section{CONCLUSION}

This study obtains the probabilities of an infinite server queues with Poisson arrivals and exponential service times when the parameters of both distributions are allowed to vary with time. The transient probabilities of the system are given by using the generating function and Bessel functions.

\section{REFERENCES}

Abl'nikov, L.M., 1968. Anonstationary queueing problem for a system with infinite number of channels for a group arrival of requests. Problem Inform. Trans., 4: 82-85.

Al Seedy, R.O. and F.M. Al-Ibraheem, 2003. New transient solution to the $\mathrm{M} / \mathrm{M} / \infty$ queue with varying arrival and departure rate. Applied Math. Comput., 135: 425-428. DOI: $\quad$ 10.1016/S00963003(02)00007-3

Bagchi, T.P. and J.G.C. Templeton, 1972. Numerical Method in Markov Chains and Bulk Queues. Springer-Verlag, Berlin, ISBN: 0387059962, pp: 89.
Clarke, A.B., 1956. A waiting line process of Markov type. Ann. Math. Stat., 27: 452-459. http://projecteuclid.org/DPubS?service=UI\&versio $\mathrm{n}=1.0 \&$ verb=Display\&handle=euclid.aoms $/ 117772$ 8268

Collings, T. and C. Stoneman, 1976. The M/M/ $\propto$ queue with varying arrival and departure rate. Operat. Res., 24: 760-773. http://www.jstor.org/pss/169773

Hasofer, A.M., 1964. On the single-server queue with non-homogeneous Poisson input and general service time. J. Applied. Problem, 1: 369-384. http://www.jstor.org/pss/3211866

Leese, E.L. and D.W. Boyed, 1966. Numerical methods of determining the transient behavior of queues with variable arrival rates. J. Can. Opnal. Res. Soc., 4: 1-13.

Saaty, T.L., 1961. Elements of Queueing Theory: With Applications. Mcgraw-Hill, New York, pp: 423. http://books.google.com.pk/books?id=z9I-

AAAAIAAJ \&q=Elements+of+Queuing+Theory, \& $\mathrm{dq}=$ Elements+of+Queuing+Theory

Shanbhag, D.N., 1966. On infinite server queues with batch arrivals. J. Applied Problem, 3: 274-279. http://www.jstor.org/pss/3212053

Takacs, L., 1955. Investigation of waiting time problems by reduction to Markov processes. Acta. Math. Hung., 6: 101-129. DOI: 10.1007/BF02021270 